\title{
Redes de gobernanza en el clúster turístico de Mazatlán
}

\author{
Governance networks \\ in Mazatlan tourism cluster
}

\author{
Miriam Nava Zazueta* (D) http://orcid.org/0000-0003-1194-4747 \\ Alejandro Mercado-Celis** (iD http://orcid.org/0000-0002-5777-6104
}

\section{Resumen}

El objetivo del artículo es demostrar que la gobernanza del clúster turístico de Mazatlán se produce en dos contextos y con lógicas distintas. En el primero intervienen actores regionales, y se reproduce un modelo de turismo tradicional; en el segundo participan actores intraurbanos, para lograr la recuperación del centro histórico y su incorporación exitosa a una oferta turística nueva, que se pretende sea para consumir la ciudad. Ambos sistemas se estudian a partir del análisis de redes, en el que se destacan las relaciones entre actores, la periodicidad y los contenidos; empero, no se determinaron los vínculos de entrada y salida entre los nodos. Un hallazgo central es que en un clúster es factible la existencia de más de un sistema de gobernanza. Se concluye que en Mazatlán, como destino turístico, la calidad de la dinámica relacional, como sistema de gobernanza, condiciona el desempeño del clúster en general y del centro histórico en particular. Palabras clave: clúster turístico; gobernanza; análisis de redes; destinos turísticos; Mazatlán.

\begin{abstract}
The aim of this article is to demonstrate that Mazatlan tourism cluster governance occurs in two contexts and according to different logics. In the first one, regional actors intervene, reproducing a traditional tourism model, whereas in the second one, intra-urban stakeholders participate. This allowed the recovery of the historic center and its successful incorporation into a new tourist offer to consume the city. Both systems are studied on the basis of network analysis, in which relations among actors, periodicity and contents are emphasized; however, input and output links between nodes were not identified. A central finding is that the existence of more of one system of governance in a cluster is feasible. It therefore follows that in Mazatlan, as a tourist destination, quality of relational dynamics, as a governance system, conditions the performance of the cluster in general and that of the historic center in particular.

Keywords: tourism cluster; governance; network analysis; tourist destinations; Mazatlan.
\end{abstract}

Cómo citar: Nava Zazueta, M., y Mercado-Celis,A. (2019). Redes de gobernanza en el clúster turístico de Mazatlán. región y sociedad, 3I, el003. doi: I0.22198/rys2019/31/I003

* Universidad Autónoma de Sinaloa, Facultad de Estudios Internacionales y Políticas Públicas. Prolongación Josefa Ortiz de Domínguez s/n, Polígono Sur, C. P. 80040. Ciudad Universitaria, Culiacán, Sinaloa, México. Correos electrónicos:mir_nava@hotmail.com; mirnava@uas.edu.mx

** Universidad Autónoma Metropolitana, unidad Cuajimalpa, Departamento de Ciencias Sociales. Avenida Vasco de Quiroga 4871, Santa Fe Cuajimalpa, C. P. 05370, Cuajimalpa, Ciudad de México, México. Autor para correspondencia. Correo electrónico: ale_mercado@yahoo.com

Recibido: 30 de octubre de 2017

Aceptado: 18 de mayo de 2018

Liberado: 23 de enero de 2019

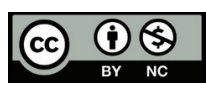




\section{Introducción}

El desempeño económico de las regiones trasciende las firmas individuales, ya que el entorno en que operan influye en sus resultados; el sistema de gobernanza es una dimensión básica del espacio económico regional, que en los destinos turísticos tiene una relevancia especial porque la región misma es el producto por vender (Nava, 2013), y también debido a que esos lugares enfrentan una competencia más acuciante para colocarse y mantenerse en el mercado, ya que el avance tecnológico permite que cada vez más lugares sean competidores potenciales. Por ello, el análisis de la gobernanza de dichos espacios cobra mayor importancia para entender su funcionamiento, desempeño y estrategias para mejorar su posición en los mercados.

El objetivo de esta investigación es demostrar que los clústers turísticos no necesariamente forman un sistema de gobernanza coherente y articulado, y que en una aglomeración pueden existir varios con acción opuesta. Para demostrarlo se analiza el clúster turístico de Mazatlán en dos contextos: uno donde los actores intervienen en el ámbito regional, y otro donde éstos inciden en el intraurbano, en específico en el centro histórico de la ciudad. Ambos sistemas de gobernanza se estudian a partir de la perspectiva de redes, se analizan las relaciones entre los actores y los elementos que las motivan.

El artículo está organizado en cinco apartados, en el primero se ubica teóricamente la discusión de los destinos turísticos como clústers y el papel de la gobernanza en su competitividad. En seguida se presenta la problemática de Mazatlán como lugar turístico en México. El tercero aborda la metodología utilizada en la investigación; el cuarto expone los resultados y por último las conclusiones.

\section{Aproximación teórica}

Es factible impulsar el desarrollo desde el ámbito local cuando se genera un proceso de gobernanza que posibilita a los actores gestionar políticas e intervenir en las que atañen a su espacio económico y de vida. En este sentido, la gobernanza es un mecanismo que refiere a las relaciones entre los actores, la manera en que interactúan entre ellos y las reglas o convenciones que regulan la interacción (Beritelli, Bieger y Laesser, 2007). Duran (2013) afirma que la gobernanza es un fenómeno social en el cual participan líderes de los sectores público y privado, y diversifican sus roles de acuerdo con sus necesidades, es decir, es un instrumento útil para conceptualizar problemas, oportunidades y contextos, así como para fijar los límites entre el Estado, los empresarios y la sociedad. Esta dinámica propicia interdependencias ya que ninguna de las partes, de manera unilateral, cuenta con toda la información y la capacidad para resolver problemas o impulsar proyectos.

La gobernanza se traslada al turismo, al indagar formas nuevas de administrar los destinos e incrementar su competitividad, en busca de asociaciones de colaboración público-privadas (Azizpour y Fathizadeh, 2016; Queiroz y Ras- 
trollo, 2015). En este tenor, Pearce (2014) argumenta que un destino turístico es un producto y a la vez una forma de producción, en el que una variedad de actores trabaja de manera conjunta para brindar un servicio al cliente. A su vez, Bieger (1998) afirma que los destinos toman la posición de productos que compiten en los mercados y requieren ser administrados como negocios, sobre todo a partir del nuevo milenio, ya que el turismo como sector económico ha tomado un rol prioritario.

En esta lógica, el trabajo pionero de Svensson, Nordin y Flagestad (2005) relacionó la gobernanza con el desarrollo de los destinos turísticos, y la tipificó como una red de interacción entre actores públicos y privados, que son interdependientes y comparten información y recursos para lograr objetivos comunes. En 2006, los autores acuñaron el concepto "gobernanza de los destinos turísticos" y, al vincularlo con los enfoques nuevos de desarrollo regional, concluyeron que uno de los retos principales en la industria es lograr una visión de futuro compartida. Aquí, el desafío del gobierno es de impulsar sinergias con los otros sectores, y así estimular un sistema de gobernanza eficiente que resulte en mejoras para el destino.

A partir de estos aportes, los destinos turísticos se han analizado con el enfoque de gobernanza. Nordin y Svensson (2007) estudian la relación entre la estructura de gobernanza y el desempeño de los destinos turísticos, se centran en las relaciones entre los sectores gubernamental y turístico, y los recursos que fluyen a través de sus redes. Por su parte, las propuestas de Beritelli, et al. (2007) y Pechlaner, Beritelli y Volgger (2015) cuestionan los modelos de gobernanza adecuados para el éxito de los espacios turísticos; toman en cuenta la historia del lugar, las asimetrías de poder y la interdependencia entre actores. Estas investigaciones enfatizan la gestión de los destinos como un modelo empresarial. A su vez, las propuestas de D’Angella y Gob (2009) hacen hincapié en incluir a todas las partes interesadas en el sistema de gobernanza, y así trascender la visión de los actores económicos y gubernamentales. Baggio, Scott y Cooper (2010) explican que un destino turístico funciona como un sistema socioeconómico complejo, en donde la gobernanza opera como mecanismo que le permite construir capacidades de aprendizaje y adaptación, y así acrecentar la eficiencia del sistema. Sin embargo, los acuerdos entre los actores y la calidad de su dinámica relacional inciden en la eficiencia del espacio, como producto turístico, y en su competitividad.

\section{Mazatlán como destino turístico}

En Sinaloa, México, Mazatlán es el principal destino, tanto en captación de visitantes como de ingresos derivados del turismo; entre 2005 y 2016 captó, en promedio a $74 \%$ de los turistas, y en el mismo periodo generó, en promedio, 88\% del total de los ingresos por la actividad (Aguilar, 2006, 2008, 2010; López, $2013,2016)$. A su vez, en el estado se ha incrementado la importancia económica del turismo, su aportación al producto interno bruto (PIB) aumentó de $11.9 \%$, en 2010, a 13.4, en 2014, y Mazatlán fue el eje (Aguilar, 2010, p. 205; López, 
2016). El clúster turístico es crucial para el municipio, constituye su industria motriz; en 2005 generó 30\% del PIB, en 2008 aumentó a 46.9 (Nava e Ibarra, 2010), y a la fecha casi no ha variado, aunque en el contexto nacional el destino tiene poca relevancia.

\section{Problemática}

Entre el año 2000 y 2010, Mazatlán se mantuvo en la cuarta posición como receptor de cruceros, así como de llegadas de vuelos internacionales regulares en destinos de sol y playa en México. Aunque en ambos rubros conservó el cuarto sitio, la brecha se amplió, y cerró la década con una contracción de $3 \%$ en el mercado de cruceros, con respecto a 2007; de 2011 a 2013 este mercado colapsó, y Mazatlán se ubicó en el octavo lugar. Para el cierre de 2016 este nicho de mercado parecía que se estaba recuperando pero aún continúa en el octavo lugar nacional como destino de cruceros, y con una brecha negativa amplia respecto a los mejor colocados (Secretaría de Turismo [SECTUR], 2017b). En la conectividad aérea, entre 2007 y 2015 las llegadas internacionales tuvieron una baja de 40\% (SECTUR 2017a), lo que indica pérdida de competitividad en este rubro.

La problemática se enfrentó con un plan municipal y otro estatal; en 2002, el gobierno local presentó el Plan Maestro de Turismo "Mazatlán XXI” cuya prioridad fue reposicionar al municipio en los mercados. Entre 2002 y 2007, el sector fue orientado conforme a las propuestas del plan y logró incrementar el número de cruceros y llegadas internacionales. En 2007, el gobierno de Sinaloa creó la Secretaría de Turismo e impulsó el Plan Estratégico de Turismo de Sinaloa (Plan Avante), para reconfigurar la posición del estado en los mercados de turismo. En él se definieron tres polos de desarrollo turístico, con Mazatlán como punto nodal de la estrategia. Sin embargo, los datos indican que han tenido pocos resultados en mejorar la competitividad respecto a otros lugares de sol y playa, que es su objetivo prioritario.

En 2014, de nuevo se realizaron planes para optimar la competitividad de Mazatlán, ese año se presentó el proyecto "Hacia un Modelo de Turismo Competitivo y Sustentable en Mazatlán y el Sur de Sinaloa"; a su vez, el gobierno federal impulsó la creación de agendas para la competitividad de los destinos turísticos en México. El gobierno estatal y la Universidad de Occidente elaboraron la "Agenda para la Competitividad de Mazatlán, 2013-2018”. Al igual que los planes diseñados antes para incrementar la competitividad del puerto, el enfoque principal de estos proyectos nuevos se centra en elementos sectoriales, infraestructura turística y urbana y recursos del ramo; sin embargo, sigue quedando al margen la participación de actores centrales, que hacen a Mazatlán un producto turístico, e inciden directamente en la experiencia que vive un visitante.

Mientras que esto sucedía en el plano gubernamental, el proceso de revitalización del centro histórico de Mazatlán empezó a tener un efecto importante para el turismo. Lo interesante es que el objetivo primario en el proceso 
de rehabilitación provino de fuentes externas al sector turístico, inclusive en contra de la voluntad de hoteleros poderosos. El impulso inicial emanó de intelectuales locales y habitantes de la zona, que pretendían recuperarlo como patrimonio de la ciudad y mejorarlo como espacio de vida. El eje central giró en torno al rescate de la infraestructura urbana para uso de los habitantes, que se empobreció y deterioró a partir de la década de 1940, al decaer la actividad comercial del puerto, y cuando empezó el abandono del centro histórico, al grado de que lentamente se fue convirtiendo en un espacio de edificios vacíos. Aunado a ello, a partir de 1960, con la transición del polo turístico del casco antiguo de la ciudad a la hoy zona dorada, el viejo Mazatlán quedó desarticulado de la oferta turística, lo que ocasionó daños colaterales como el deterioro de las fincas abandonadas del centro histórico, que poco a poco quedó en ruinas a pesar de su valor patrimonial. Así, una de las zonas principales del puerto quedó en el olvido y se fue deteriorando hasta el punto de considerar la demolición como alternativa, para optimizar el uso del suelo y darle plusvalía a la zona, aunque se dejara de lado su valor patrimonial y cultural (Alvarado, 2007).

En 1972, los agremiados de la Cámara Nacional de Comercio gestionaron ante las instancias municipales la restauración del teatro Ángela Peralta, su objetivo era ampliar la oferta turística, y dotar a la sociedad mazatleca de un espacio cultural, pero no existió un plan definido. En 1974, con la constitución del Patronato Pro Restauración del Teatro Ángela Peralta comenzaron los esfuerzos de la sociedad por restaurar y preservar los edificios del centro histórico, para dotar a la localidad de un entorno cultural de esparcimiento que recordase su antiguo esplendor. El esfuerzo del patronato tuvo resultados hasta 1980, cuando los vecinos pugnaron por la restauración de la zona y se conformó un sistema de gobernanza entre ellos y las autoridades, para la recuperación de este patrimonio histórico.

En 1987 el presidente municipal, José Ángel Pescador, dio a conocer el Programa de Rescate, Revitalización y Conservación del Patrimonio Nacional del Centro Histórico de Mazatlán. En él se indicaban tres ejes, y la meta era incorporar el sitio a la dinámica económica de la localidad y, a su vez, regresarle su antiguo esplendor para venderlo como lugar turístico. Las inversiones empezaron a fluir, así como las inconformidades. Aun así, la autoridad local, en un trabajo conjunto con el Patronato Pro Restauración del Teatro Ángela Peralta, mantuvo las inversiones y sostuvo la tesis de que "Mazatlán es algo más que turismo de sol y playa" (Urquijo, R., comunicación personal, director de Desarrollo Turístico. Secretaría de Turismo de Sinaloa, 3 de mayo de 2010 y 11 de junio de 2010).

En el sexenio de Francisco Labastida Ochoa (1987-1992) se concretó el trabajo del sistema de gobernanza impulsado por los vecinos del centro histórico y el municipio, al lograr que el gobierno federal destinara 1400 millones de pesos al rescate del teatro. El patronato, conformado por vecinos de la zona, intelectuales y académicos aportó trabajo y dinero para la restauración (Santamaría, 2007). El Ángela Peralta dio su primera función el 5 de diciembre de 1987, en pleno proceso de restauración. El 9 de diciembre de 1990 fue declarado Monumento Histórico y Patrimonio de la Nación, y el 23 de octubre de 1992 fue rein- 
augurado por el entonces presidente Carlos Salinas. Así se concretó el objetivo que, desde 1970, se propuso el patronato que pugnó por su restauración. Este sistema de gobernanza intraurbano logró, a través del impulso bottom-up, un proceso de recuperación, y sentó las bases para la revitalización económica y de entretenimiento en la zona, elementos fundamentales para su incorporación posterior a la oferta turística.

A mediados de la década de 1990 el teatro empezó a funcionar, y se creó el Centro Municipal de Artes, a un costado de este inmueble restaurado, y gracias al mejoramiento urbano se instalaron negocios pequeños alrededor. La primera etapa fue difícil, la afluencia de personas era insuficiente para dinamizar económicamente el área, pero poco a poco se convirtió en un espacio de entretenimiento para locales y extranjeros. En 1997, los hermanos Gómez-Rubio inauguraron el primer restaurante, "Pedro y Lola", frente a la plazuela Machado, con un estilo que concordaba con el ambiente cultural y de antaño del Mazatlán antiguo; esta apertura marcó el principio de la transición del centro histórico, como espacio de vida, a uno para el turismo. Un año después, la misma familia inauguró el hotel boutique The Melville. A partir de la intervención de los hermanos Gómez-Rubio se tuvo una visión más clara para darle más uso al centro histórico. En 2002 nació la Asociación Proyecto Centro Histórico A. C., con la meta de revitalizar económica y socialmente la zona. A partir de ello se inició una etapa nueva en el proceso de recuperación del casco antiguo, y se generó un sistema de gobernanza intraurbana, pues aquél creado para recuperar el teatro había cumplido su finalidad.

\section{Metodología}

La investigación cuenta con dos unidades de análisis: a) el sistema de gobernanza del clúster turístico de Mazatlán a escala regional, considerado así en virtud de que en él participan actores locales, estatales y federales (ver Tabla 1) y b) el sistema de gobernanza intraurbano, del centro histórico de la ciudad, que toma en cuenta el proceso de rehabilitación del área, y su revitalización e incorporación a la vida económica de la ciudad, a través de la industria turística. Este último se identificó parcialmente en estudios previos (Nava, 2013; Nava y Valenzuela, 2014), y con mayor profundidad en esta investigación, al desagregar la densidad y la cohesión de la red de gobernanza regional.

El sistema de gobernanza regional se analizó a partir de su red completa de actores, organizados en una estructura que muestra las interacciones entre las partes. Los datos se obtuvieron de la encuesta sobre dinámica relacional, aplicada a 27 actores territoriales en el clúster turístico de Mazatlán, que fueron centrales e incidieron en la conformación del destino como producto turístico (ver Tabla 1). La información recopilada refiere a las estrategias para mejorar la competitividad del destino, utilidad de la información compartida, temporalidad de reuniones para gestión de recursos, mejora de conectividad aérea y de infraestructura urbana. Los datos sobre las relaciones se procesaron con el programa UCINET 6 para Windows, el cual permite identificar las característi- 


\section{Tabla 1. Actores territoriales principales del clúster turístico de Mazatlán}

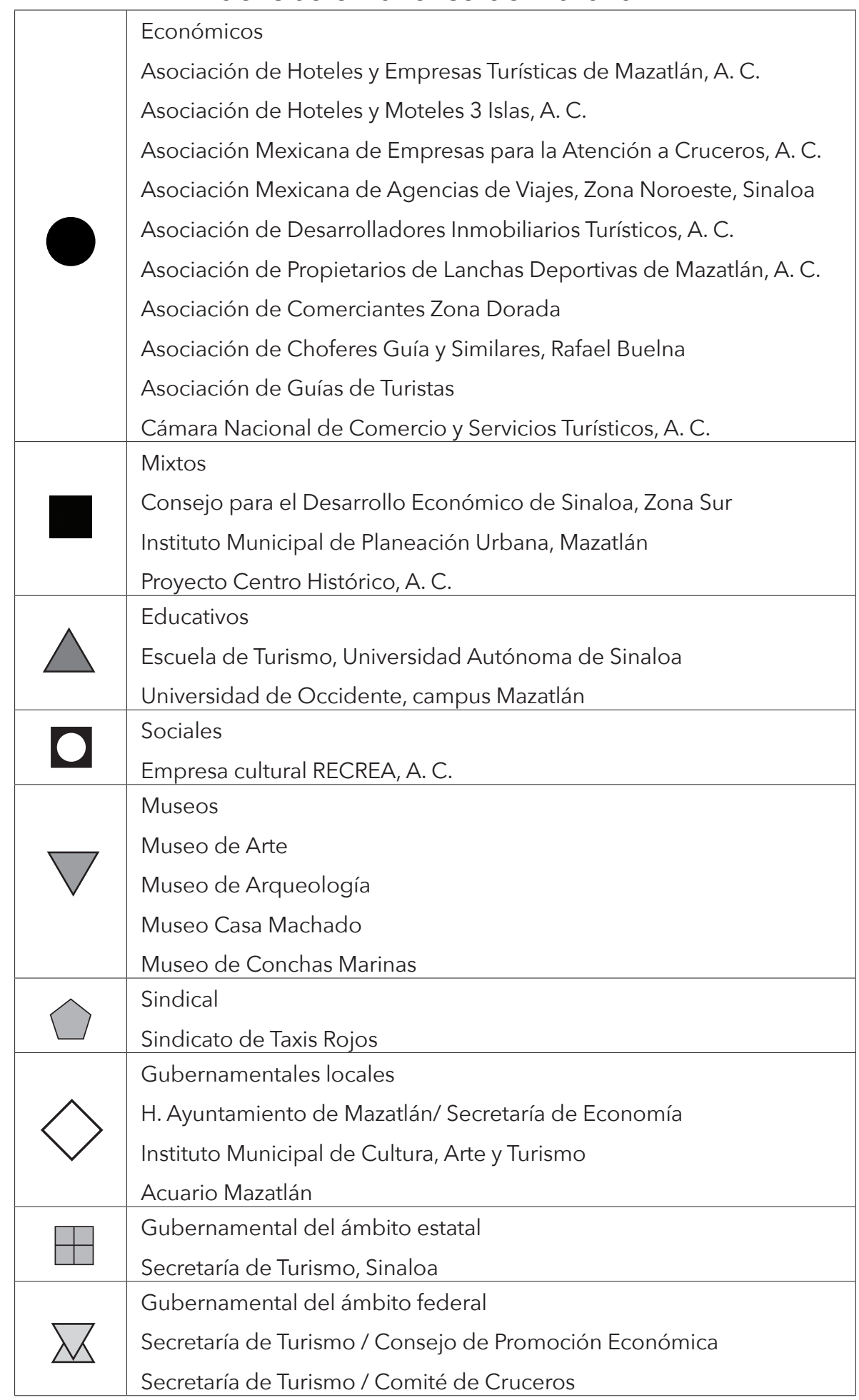

Fuente: elaboración propia, con base en información obtenida de informantes clave con método nomilalista. 
cas estructurales en las redes (Borgatti, Everret y Freeman, 2002). El análisis se realizó a partir del método de redes completas, que incluyó al censo de los actores y todas las relaciones posibles entre ellos; se identificó la existencia de la relación, y también la frecuencia para determinar la calidad del sistema de gobernanza, al indicar a quienes detentan la cohesión real en el destino, de acuerdo con su periodicidad de comunicación. Con los datos de la matriz se obtuvieron los indicadores fundamentales en los análisis de redes: los que estudian las medidas de centralidad sobre los nodos y la estructura formal de la red. En el caso de esta última, para ambas redes se utilizó densidad de la red (o cohesión), definida como la proporción existente de todos los vínculos posibles de presentarse en el sociograma (Wassermann y Faust, 1994). Este indicador se usa para analizar la calidad del sistema de gobernanza del destino, ya que constituye uno de los principales al estudiar la realidad social, y permite comparar las formas en que se comporta el colectivo; si su valor es aproximado a la unidad significa que es una red cohesionada, y si es cercano a cero indica distancia relacional.

Como la densidad de una red se calcula sin considerar la frecuencia de contacto, el indicador se complementó con la distribución de densidad o cohesión, la cual indica a quienes detentan la mayor cohesión respecto a su frecuencia de comunicación; los vínculos que se realizan con más frecuencia indican el mayor dinamismo en el sistema de gobernanza y, por ende, a los actores que participan más en la construcción del destino y la preponderancia de sus decisiones. Mientras que el indicador de densidad refiere a la estructura reticular, las medidas de centralidad permiten analizar la posición de los nodos (actores) en ella, e identificar la importancia de cada uno en virtud de su cercanía e influencia con sus contrapartes del sistema de gobernanza. La centralidad considera tres indicadores: rango, grado de cercanía y grado de intermediación.

El rango indica el número de vínculos directos que tiene un actor, y es proporcional a la influencia de éste en el sistema, su capacidad para tener acceso a la información, y mayor importancia en la toma de decisiones. El grado de cercanía refiere a la proximidad de un nodo respecto al resto de la red, es decir, su capacidad para tener contacto con sus contrapartes, de manera directa o a través de un tercero. El grado de intermediación indica cuando un actor es puente entre dos o más partes del sistema, que no tienen vínculos entre sí.

El sistema de gobernanza vinculado con el centro histórico presenta su análisis en dos temporalidades; la primera explica la rehabilitación de la zona, a partir de información histórica y empírica, y la segunda analiza y explica el sistema intraurbano, a partir de los actores del clúster turístico, cuyo dinamismo influye en la revitalización de la zona, para dotarla con atractivos materiales e inmateriales que la incorporan al ámbito turístico, con lo que se diversifica la oferta tradicional relacionada con sol y playa.

Para obtener la información empírica regional se utilizó un censo de la Secretaría de Turismo sobre las principales asociaciones e instituciones ligadas a la industria. Se comparó y complementó con los datos recabados y así se integró el censo final de actores, que se ubicaron en cinco rubros: económicos, guber- 
namentales, sociales, educativos y mixtos, estos últimos conformados por la iniciativa privada organizada, la sociedad civil y el gobierno, y sus interdependencias se establecieron como elementos explicativos de la posición competitiva del destino en los mercados (Merinero, 2015; Nava, 2013).

La información empírica se recopiló entre 2011 y 2016. Primero se realizó el censo de actores territoriales y luego se diseñó, piloteó y aplicó la encuesta a todos, lo que permitió la construcción del sociograma del sistema de gobernanza regional. Para comprender mejor el objeto de estudio, se realizó observación no participante, a través de visitas reiteradas al destino, para evitar sesgos cada observador hizo su registro y después se contrastaron.

Para identificar el sistema de gobernanza intraurbano se recurrió al análisis histórico y aplicación de ocho entrevistas semiestructuradas, en 2013 y 2014, a actores clave en la rehabilitación del centro histórico. Al compaginar la información documental con la empírica, se identificó el sistema de gobernanza intraurbano, que sirvió como punto de partida para la revitalización del área, y después para identificar y analizar al sistema de gobernanza actual, que dinamiza la zona. Por último, para reforzar el análisis, se levantó un censo de unidades económicas y culturales vinculadas con el turismo en el área, para determinar si el proceso de revitalización se engarzó con la industria motriz de Mazatlán.

Respecto a las limitantes de la investigación, no se determinaron los vínculos de entrada y salida entre los actores, sino la existencia y temporalidad de la relación, así como sus contenidos; por otro lado, los intereses nuevos entre las partes pueden modificar la estructura de la red de gobernanza, y propiciar mayor o menor cohesión e inclusive la creación de redes con intereses particulares, que no necesariamente beneficien al clúster como producto turístico.

\section{Resultados}

Los resultados se presentan en dos vertientes, la primera explica el sistema de gobernanza del clúster turístico, su dinamismo e incidencia en la proximidad (o distancia) relacional existente en el destino, dilucidación que se vincula con su posición competitiva en los mercados. Asimismo, se analiza y explica quiénes son los actores principales del sistema, los rubros que los cohesionan y cómo influyen en el desempeño del lugar. La segunda explica el sistema de gobernanza que permitió la recuperación del teatro Ángela Peralta, y empezó la revitalización del centro histórico convirtiéndolo en espacio icónico y parte fundamental de la oferta turística.

\section{Gobernanza global del clúster y sus actores centrales}

El primer elemento de análisis en la red es el sistema regional de gobernanza, indicado como cohesión global de la conectividad entre sus actores territoriales, esto es, los vínculos totales sin considerar la periodicidad con que se lleva 
a cabo. La estructura de gobernanza del clúster turístico está conformada por una red de 27 actores cuya cohesión global es de $48.4 \%$, es decir, conciben 340 vínculos de los 702 posibles de generarse (ver Tabla 2 y Figura 1), e indica que la distancia relacional es casi igual al grado de cohesión.

Tabla 2. Intensidad institucional general en el clúster turístico de Mazatlán

\begin{tabular}{|l|c|c|}
\hline Frecuencia de contacto & Vínculos & Porcentaje respecto al total \\
\hline Vínculos posibles en la red & 702 & 100 \\
\hline Global & 340 & 48.4 \\
\hline Diario & 14 & 2.0 \\
\hline Semanal & 54 & 7.7 \\
\hline Mensual & 48 & 6.9 \\
\hline Semestral & 112 & 15.9 \\
\hline Anual & 112 & 15.9 \\
\hline Nunca & 362 & 51.60 \\
\hline
\end{tabular}

Fuente: elaboración propia, a partir de Nava-Zazueta (2012).

Otros estudios en lugares turísticos muestran que a mayor cohesión existe más efectividad, siempre y cuando ésta no disminuya drásticamente al acotar la frecuencia de comunicación. Esto es, a pesar de existir distancia relacional en el sistema, si el número de vínculos se conserva en una periodicidad de preferencia entre un día y un mes, es factible que el sistema de gobernanza sea eficiente en el impulso de mejoras en el destino. Empero, si la cohesión es alta, pero la comunicación es distante (una vez cada seis meses o más) es difícil que sea eficaz para mejorar el desempeño; este hecho permite contradecir la tesis tradicional de que mayor densidad relacional implica más competitividad, pues es imprescindible la densidad de vínculos, la temporalidad en que se realizan y las acciones que los motivan, como lo muestran los estudios de Prats, Guia y Molina (2008), en Costa Brava; Scott, Cooper y Baggio (2008), en Queensland; Baggio, Scott y Copper (2010), en Elba; Merinero (2010), en Écija, y Nava (2013), en Mazatlán. Así, el análisis del primer indicador del sistema regional de gobernanza permite vislumbrar que ésta es ineficiente por la distancia relacional entre sus actores (ver Tabla 2). 
Figura 1. Sistema de gobernanza regional en el clúster

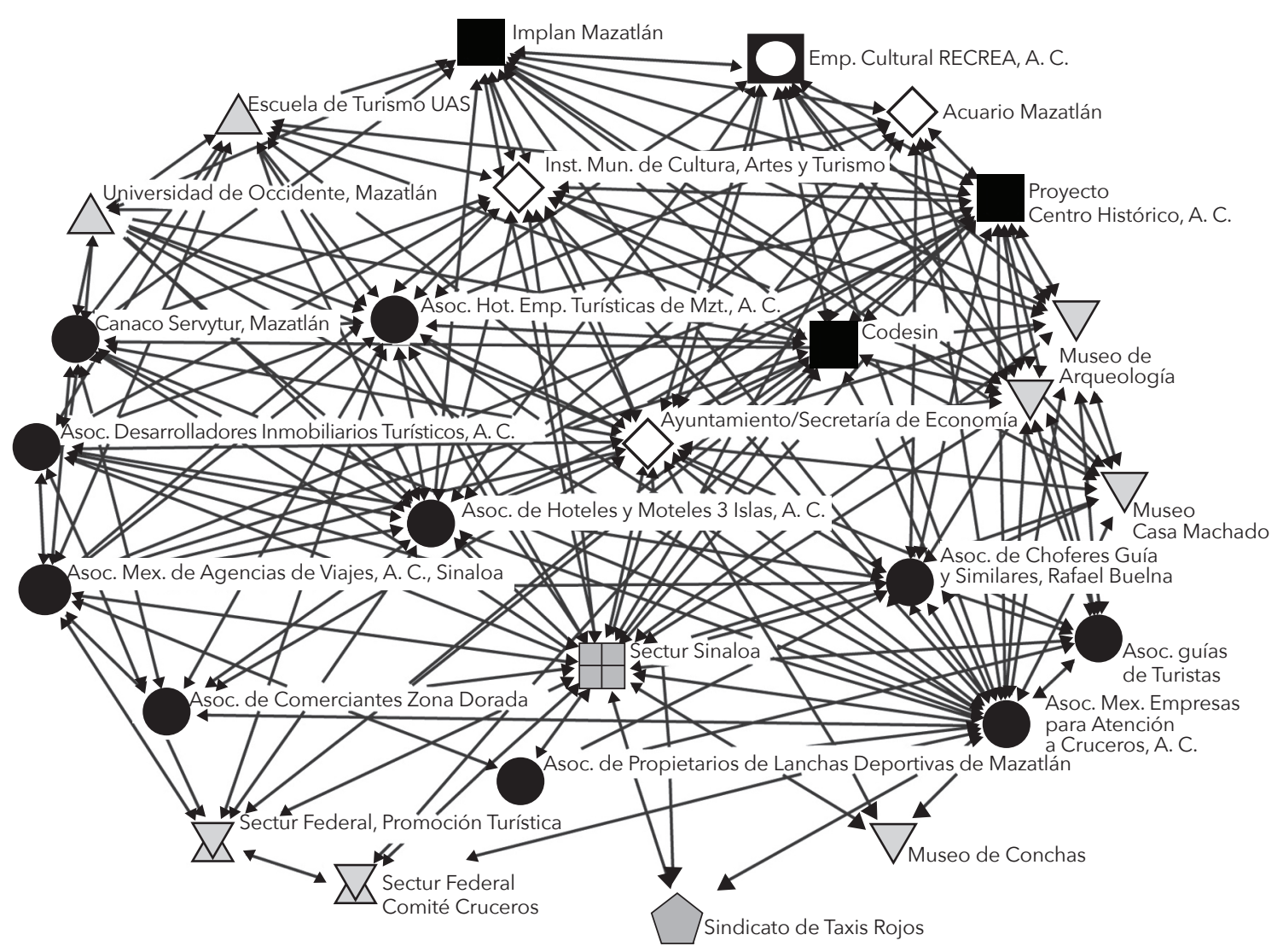

Fuente: elaboración propia, a partir de Nava-Zazueta (2012).

Respecto al análisis de la red global de gobernanza del clúster, la Figura 1 ilustra el sociograma general del sistema, y permite identificar a los actores de mayor dinamismo. La red está construida a partir de lazos bidireccionales y destacan la SECTUR, Sinaloa, la Asociación Mexicana para la Atención a Cruceros, A. C., y la Secretaría de Economía del Ayuntamiento, como los actores que están vinculados con casi todas sus contrapartes; son los mejor posicionados en cuanto a disposición de información, de ahí la posibilidad de que puedan influir en el clúster e incluso servir de puente entre ambos sistemas de gobernanza. En el centro inferior del gráfico se observa que SECTUR Sinaloa tiene más vínculos, lo que indica que tiene el mayor grado de accesibilidad en la red y a la información que fluye a través de ella. Además, tiene el grado máximo de cercanía al clúster, con 100 puntos; su intermediación o capacidad para servir de puente entre los actores que no tienen vínculos entre sí es de 100\%, lo que indica que es el actor mejor vinculado y con mayor capacidad de negociación en el sistema, para impulsar proyectos integradores e inclusive para aislar actores (ver Tabla 3). 
Tabla 3. Indicadores de centralidad del sistema de gobernanza regional del clúster turístico de Mazatlán

\begin{tabular}{|c|c|c|c|}
\hline Actor & Rango & $\begin{array}{l}\text { Grado de } \\
\text { cercanía }\end{array}$ & $\begin{array}{l}\text { Grado de } \\
\text { intermediación }\end{array}$ \\
\hline Secretaría de Turismo de Sinaloa & 26 & 100.0 & 100.0 \\
\hline $\begin{array}{l}\text { Asociación Mexicana Empresas para Atención } \\
\text { a Cruceros, A. C. }\end{array}$ & 25 & 96.3 & 85.0 \\
\hline Secretaría de Economía/Ayuntamiento & 23 & 86.7 & 50.6 \\
\hline Consejo para el Desarrollo Económico de Sinaloa, Zona Sur & 18 & 76.5 & 20.9 \\
\hline Proyecto Centro Histórico, A. C. & 18 & 76.5 & 17.5 \\
\hline Asociación de Hoteles y Moteles 3 Islas, A. C. & 17 & 74.3 & 12.7 \\
\hline Instituto Municipal de Cultura, Artes y Turismo & 17 & 74.3 & 11.3 \\
\hline Asociación Mexicana de Agencias de Viajes, A. C., Sinaloa & 16 & 72.2 & 16.6 \\
\hline $\begin{array}{l}\text { Asociación de Hoteles y Empresas } \\
\text { Turísticas de Mazatlán, A. C. }\end{array}$ & 16 & 72.2 & 11.3 \\
\hline Instituto Municipal de Planeación Urbana Mazatlán & 15 & 70.3 & 7.5 \\
\hline Escuela de Turismo, Universidad Autónoma de Sinaloa & 13 & 66.6 & 2.0 \\
\hline $\begin{array}{l}\text { Cámara Nacional de Comercio } \\
\text { y Servicios Turísticos, Mazatlán }\end{array}$ & 12 & 65.0 & 1.9 \\
\hline Museo de Arqueología & 12 & 65.0 & 3.0 \\
\hline Museo de Arte & 12 & 65.0 & 3.0 \\
\hline Museo Casa Machado & 12 & 63.4 & 1.2 \\
\hline Universidad de Occidente, campus Mazatlán & 11 & 63.4 & 0.4 \\
\hline Empresa cultural RECREA, A. C. & 11 & 63.4 & 1.8 \\
\hline Asociación de Choferes Guía y Similares, Rafael Buelna & 11 & 63.4 & 7.8 \\
\hline Asociación Guías de Turistas & 10 & 59.1 & 2.2 \\
\hline Acuario Mazatlán & 10 & 60.5 & 1.7 \\
\hline Asociación de Desarrolladores Inmobiliarios Turísticos, A. C. & 9 & 61.9 & 2.8 \\
\hline $\begin{array}{l}\text { Secretaría de Turismo federal, } \\
\text { Consejo de Promoción Turística }\end{array}$ & 8 & 59.1 & 3.1 \\
\hline Asociación de Comerciantes Zona Dorada & 7 & 57.8 & 0.0 \\
\hline $\begin{array}{l}\text { Asociación de Propietarios de Lanchas Deportivas } \\
\text { de Mazatlán }\end{array}$ & 5 & 55.3 & 0.4 \\
\hline Secretaría de Turismo federal. Comité de Cruceros & 4 & 54.2 & 0.3 \\
\hline Museo de Conchas Marinas & 3 & 53.1 & 0.0 \\
\hline Sindicato de Taxis Rojos & 3 & 53.1 & 0.0 \\
\hline Media & 12.7 & 67.7 & 13.4 \\
\hline
\end{tabular}

Fuente: elaboración propia, a partir de Nava-Zazueta (2012). 
Figura 2. Sistema de gobernanza regional, temporalidad mensual

Asociación de Propietarios de Lanchas Deportivas

de Mazatlán

$\triangle$ Esc. Turismo UAS

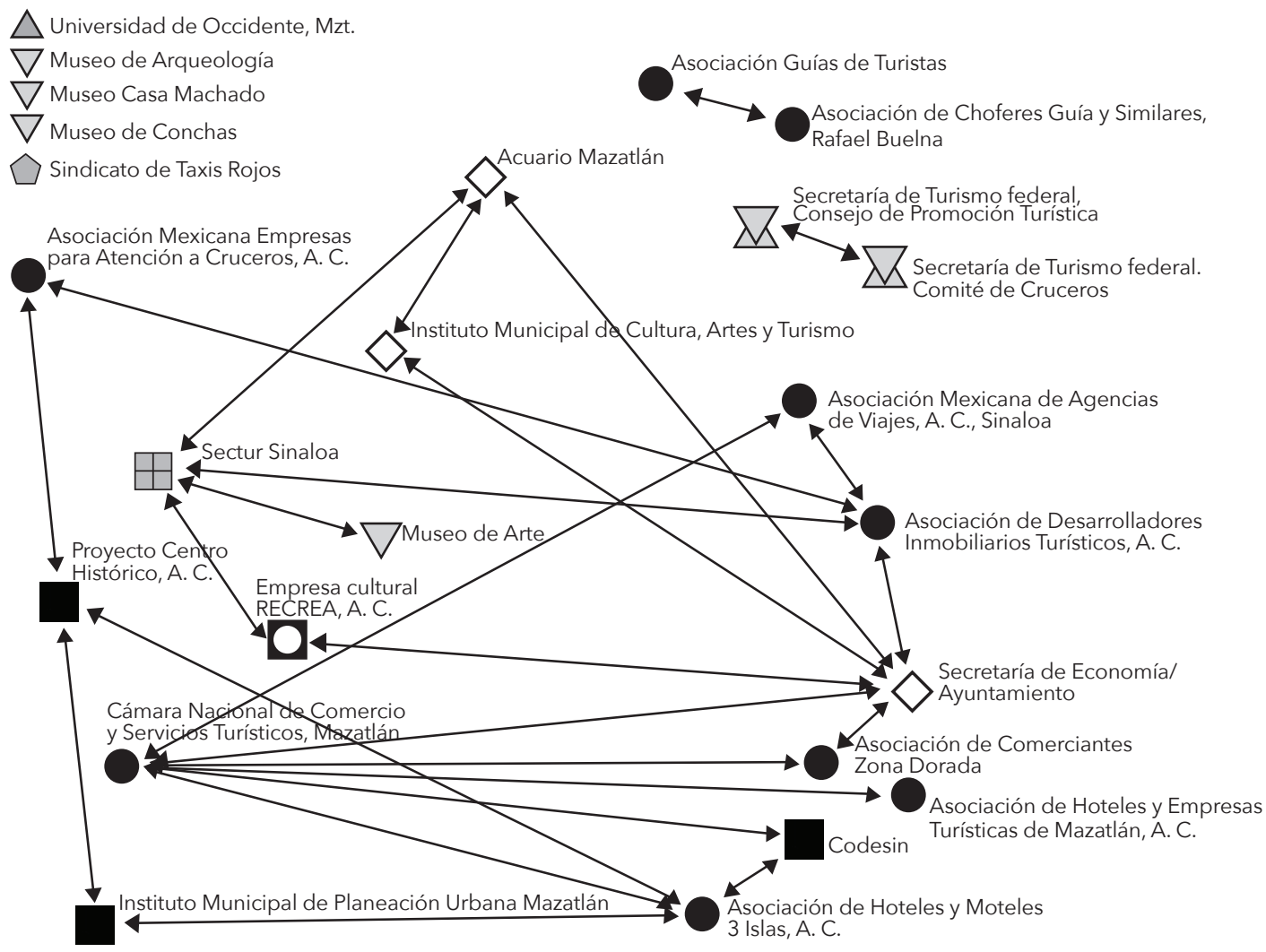

Fuente: elaboración propia, a partir de Nava-Zazueta (2012).

Que la SECTUR ostente mayor rango y los indicadores de centralidad más altos permite suponer la existencia de un sistema de gobernanza top-down. Aunque no se considera así, ya que en México, en el ámbito del turismo, la estructura top-down refiere a políticas que emanan de la autoridad central, a través de la Secretaría de Turismo federal, y en Mazatlán, la SECTUR Sinaloa constituye un actor local en virtud de que en conjunto con otros que también lo son, planea y organiza las directrices en el clúster, como lo indica la periodicidad de comunicación mensual y semanal, así como los contenidos relacionales (ver Figura 2). Por otro lado, si bien el grado de cohesión en el destino es de $48.4 \%$, al desagregarla en periodicidad, la densidad se contrae y se amplía la distancia relacional, que de origen es $51.6 \%$, en el sistema de gobernanza regional.

En el sistema de relaciones del clúster turístico de Mazatlán a mayor cercanía en la periodicidad de comunicación, disminuye la proporción de vínculos 
que se gestan en el destino. Esto sugiere carencia de participación de los actores principales en los proyectos comunes a mediano o largo plazo, que para concretarse implican vínculos frecuentes e intercambio constante de información, lo que a su vez propicia confianza entre las partes y coadyuva a mejorar la posición competitiva del destino (Nava, 2013; Watkins y Bell, 2002). Al analizar la red de gobernanza regional en el clúster turístico, en sus escalas de temporalidad (para conocer los cambios en su grado de cohesión), se encontró que de los 340 nexos, 224 se producen al menos una vez cada seis meses. Es decir, de $100 \%$ de las interrelaciones en el clúster, 65.88 tienen una periodicidad entre seis meses y un año, lo cual pone de manifiesto la distancia relacional que existe en el destino, y explica por qué no ha logrado revertir las deficiencias que subyacen en él, para mejorar su posición en los mercados. Aunado a ello, cuando se analizó la red de gobernanza regional desde la perspectiva de una red ideal para 27 actores, se encontró que en la periodicidad de comunicación menor a seis meses, en el sistema existe una distancia de $83.4 \%$, ya que se generan 116 flujos de información.

Los datos exhibidos indican que si bien la cohesión global del sistema de gobernanza regional es alta, se contrae drásticamente conforme disminuye la frecuencia de comunicación y se definen intereses comunes entre los actores, y se evidencia una distancia relacional superior a $80 \%$. En la tabla 2 se observa que la comunicación más cercana entre los actores es una vez por semana. Sin embargo, en ella solo participan 6 de los 27, y los flujos de información representan $2 \%$. Aunado a ello, el elemento principal que los aglutina en esta periodicidad refiere a la gestión de recursos para marketing tradicional. Los actores que tienen comunicación al menos una vez por semana son del ámbito económico y gubernamental. Otro elemento que indica un sistema de gobernanza poco eficiente para dinamizar el clúster tiene que ver con que 16 de los 27 actores están por debajo de la media, en lo que refiere a indicadores de centralidad y participación en la construcción de estrategias para mejorar la competitividad del destino, eso evidencia una distancia relacional entre los elementos principales que conforman a Mazatlán como producto turístico (ver Tabla 3). Estos datos de las relaciones a nivel micro (actores) se demuestran en la distancia relacional del sistema de gobernanza del clúster.

El análisis del sistema a nivel micro permite identificar que el dinamismo en el sistema de gobernanza del clúster lo propician los actores gubernamentales y las organizaciones económicas que aglomeran a las firmas turísticas. Si bien todos los elementos que inciden en la conformación del destino como producto turístico se consideran como parte del sistema, tal como indican los estudios de Beaumont y Dregde (2010) y Caffyn y Jobbins (2003); en Mazatlán, la información indica que actores imprescindibles como universidades, trasportistas terrestres, asociaciones de lanchas deportivas y museos, entre otros, están prácticamente fuera de él. Los nexos con sus contrapartes quedan difusos en el tiempo, así como su participación en las políticas que guían en el desarrollo.

Aunque los indicadores de centralidad de la Universidad de Sinaloa se ubican justo en la media (ver Tabla 3), al analizar su dinamismo en el sistema se encontró que sus nexos en mayor medida se realizan en seis meses a un año, y su contenido indica que se refieren a convenios para la prestación de servicio 
social de sus educandos, que se convierten en mano de obra barata - o gratuita- para las empresas e instituciones.

Entonces, la dinámica de gobernanza del clúster turístico de Mazatlán a escala regional sigue ligada a formas convencionales de los destinos poco exitosos. Los actores con mayor dinamismo son los que perciben un beneficio o una recompensa directa del mejor desempeño de la industria. Esto es, el ámbito gubernamental para presentar mejores indicadores a los gobernados, y el sector empresarial directamente vinculado con el turismo; de ahí la dificultad para engarzar propuestas que reviertan las deficiencias que subyacen en el destino.

Por otro lado, a pesar de que el proceder de los actores principales, ligados a la industria turística, indica poca proclividad a propuestas innovadoras para reubicar a Mazatlán en los mercados turísticos, el análisis de su dinámica relacional también permitió identificar la existencia de un sistema de gobernanza intraurbano eficiente, originado en actores desvinculados del turismo. Sin embargo, con su ímpetu construyeron una opción bottom-up de revitalización urbana, comercial y de entretenimiento en el centro histórico de Mazatlán. De manera colateral, el proceso de revitalización benefició el desempeño del destino como producto turístico, al brindarle la oportunidad de diversificar su oferta e incursionar en nuevos nichos de mercado. En la actualidad, este sistema intraurbano incorpora a los actores más dinámicos a la gobernanza regional, esto se debe a su propia evolución y a su incorporación como parte de la oferta turística de Mazatlán. Empero, el centro histórico continúa siendo un espacio des-diferenciado (Nava, 2017), es decir, que si bien no se impulsó exclusivamente como turístico, se convirtió en un atractivo, porque los residentes locales lo hacen interesante para el visitante.

\section{La gobernanza del centro histórico de Mazatlán: dinámica relacional e incorporación a la oferta turística}

La incorporación del casco antiguo de Mazatlán a la dinámica de la urbe lo reposicionó como "centro" de actividades económicas y de reunión social (Alvarado, 2007). En la última década, la gobernanza que subyace en la rehabilitación del centro histórico se modificó e incorporó a actores nuevos; entre los que se suman al sistema de gobernanza intraurbano e inclusive se posicionan como líderes en propuestas, están la empresa cultural RECREA, A. C., el Proyecto Centro Histórico, A. C., el Consejo para el Desarrollo Económico de Sinaloa, la SECTUR Sinaloa, la Asociación Mexicana de Empresas para la Atención a Cruceros y la Secretaría de Economía local. Empero, hasta el momento su impulso deriva más de gestiones para realizar acciones en concreto, que en una planeación y división del trabajo para lograr objetivos colegiados a mediano o largo plazo. El propósito que cohesiona al sistema de gobernanza intraurbano es ampliar y mejorar el área en recuperación y revitalización, aunque por razones diferentes. Mientras que los actores del ámbito social pugnan por el mejoramiento del centro histórico, para elevar la calidad de vida de sus habitantes, los gubernamentales y económicos pretenden aumentar la plusvalía de la zona 
y también el número de negocios, para convertirla en un lugar más atractivo para el turismo y con rentabilidad económica para la ciudad.

En cuanto a las gestiones por seguir mejorando el casco antiguo, el sistema de gobernanza intraurbano trabaja, de manera puntual, por enfoques o concreción de objetivos, y así logra más resultados. El factor principal que mueve al sistema es la gestión para mejorar las vialidades y el alumbrado público. Por ello, la gobernanza se analiza a partir de este rubro, y será el punto de contraste respecto al sistema de gobernanza regional del clúster. Son equiparables en virtud de que ambos están localizados en Mazatlán y sus objetivos son mejorar el destino como producto turístico.

La periodicidad de los vínculos para gestionar recursos en el rubro es mensual, y asisten 17 actores, por lo que solo éstos se consideran como parte del sistema de gobernanza intraurbano; al analizar la red que la ilustra reencontró que su cohesión asciende a 38\%. De los 272 vínculos por generarse en una red de 17 nodos, se producen 103. En términos generales, la red intraurbana indica una distancia relacional mayor a la detectada en la de gobernanza regional. Pero si se compara en su misma periodicidad, la red de gobernanza intraurbana tiene mejor desempeño que la vinculada con el sistema regional del clúster, cuya cohesión es de 48.4\%, sin considerar la periodicidad de comunicación, pero al ubicarla en los vínculos que se realizan al menos una vez al mes, se contrae a $6.9 \%$. Inclusive si se toman en cuenta todos los nexos con periodicidad de 1 a 30 días, la cohesión es de 16.6\% (ver Tabla 2), menor que la resultante del sistema de gobernanza intraurbano, ubicada en $38 \%$.

Los datos corroboran la tesis de Giuliani (2010), quien afirma que las propiedades estructurales de las redes influyen en la calidad del desempeño económico y la concatenación de objetivos, hecho que es evidente en el lento, pero exitoso, proceso de revitalización del casco antiguo donde, de 1989 al año 2000, se instalaron 13 entidades vinculadas con la economía de la experiencia, y subsistían siete que operaban desde el antiguo esplendor del centro histórico, como punto nodal. Desde principios de 2015, en el circuito existen 107 entidades económicas y/o culturales, que hacen del centro histórico un espacio emblemático de entretenimiento en Mazatlán (Nava, 2017). Los datos indican que el sistema de gobernanza intraurbano ha sido exitoso en el logro de sus metas.

En cuanto al rango, al igual que en la gobernanza regional, la SECTUR Sinaloa detenta los vínculos más numerosos, así como en los grados de cercanía e intermediación. Los datos indican un tipo de gobernanza top-down, pero no es así; su función es gestionar ante las instancias del gobierno federal, y canalizar las propuestas generadas en el ámbito local. Respecto al resto de los actores con mayor influencia en el sistema intraurbano están las asociaciones vinculadas con la industria turística, esto en virtud del reconocimiento que le dan al centro histórico como parte fundamental de la oferta del destino, y que los rubros económicos que se gestionan no se contraponen con los destinados directamente al sector turismo. Es decir, se accede a otros fondos que al mejorar la infraestructura urbana de Mazatlán, también lo hacen con su imagen y calidad. No obstante, si bien estos actores económicos aparecen como los que detentan la mayor comunicación e influencia, el liderazgo respecto a lo que "debe" ser 
Figura 3. Dinámica de cooperación en el sistema de gobernanza intraurbano en la gestión de recursos para vialidades y alumbrado para el centro histórico

Asociación de Propietarios de Lanchas Deportivas

de Mazatlán

$\triangle$ Esc. Turismo UAS

$\triangle$ Universidad de Occidente, Mzt.

$\checkmark$ Inst. Municipal de Cultura, Artes y Turismo

$\nabla$ Museo de Arqueología

$\nabla$ Museo de Arte

Asociación Mexicana de Agencias

$\nabla$ Museo Casa Machado

$\nabla$ Museo de Conchas

$\checkmark$ Acuario Mazatlán

$\$ Sectur Federal Comité Cruceros

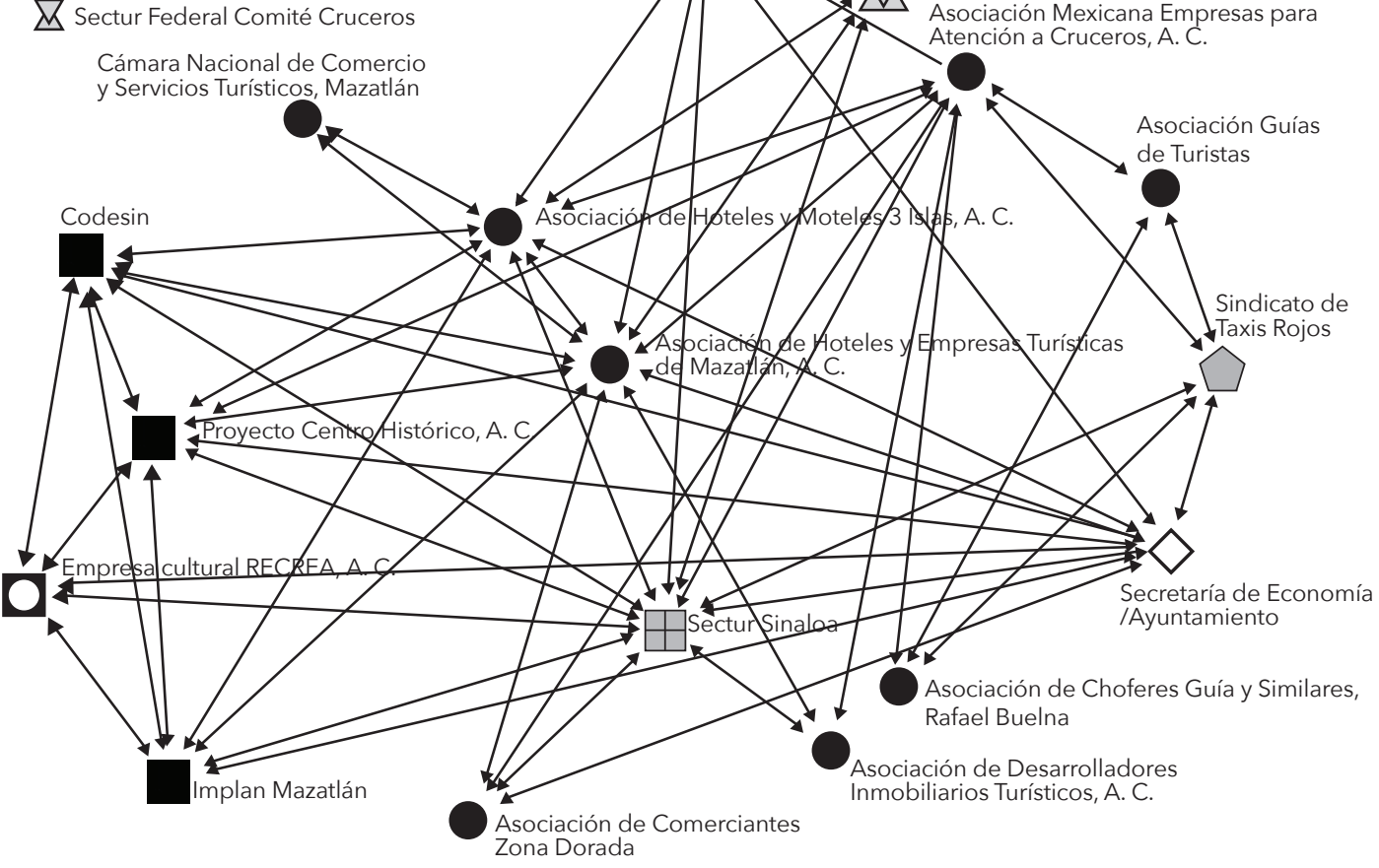

Fuente: elaboración propia, a partir de Nava-Zazueta (2012).

el centro histórico emana de las ideas originales de quienes impulsaron la recuperación y revitalización de la zona. Éstas quedaron plasmadas en la simiente de la asociación civil Proyecto Centro Histórico, A. C., fundado en 2002, para dar continuidad a estas inquietudes, y después la empresa cultural RECREA se sumó al nuevo sistema de gobernanza intraurbano que impulsa el mejoramiento del casco antiguo. Ambos se han consolidado como los promotores principales en la diversificación de las actividades para mejorar la zona, y han presionado por conseguir alumbrado artístico, adoquinado de banquetas, pintada de fachadas de inmuebles abandonados, con lo que se logró un ambiente urbano homogéneo. 
Si bien las administraciones locales habían mostrado un interés laxo en la importancia del centro histórico como parte fundamental de la oferta turística, la de 2011-2013 gestionó con éxito recursos para terminar las obras inconclusas de infraestructura, en respuesta a las peticiones reiteradas que recibió de la Empresa Cultural RECREA, el Proyecto Centro Histórico A. C., la Secretaría de Turismo, la Asociación Mexicana de Empresas para la Atención a Cruceros, las asociaciones de hoteleros y los ciudadanos involucrados. En este punto, la función de la SECTUR Sinaloa fue fundamental pues engarzó ambos sistemas de gobernanza, y logró recursos para beneficio de la ciudad y el destino. El resultado fue un aspecto nuevo, se readecuó el espacio peatonal a las necesidades del turista y del habitante local. Este último elemento es primordial en virtud de que un atractivo del casco antiguo es que permite al visitante experimentar al centro histórico como lugar de vida, un espacio en construcción permanente, pues se modifica no solo al ritmo que cambia la industria turística, sino conforme lo hace la sociedad que lo habita.

\section{Conclusiones}

La metodología de análisis de redes sociales, combinada con entrevistas y observaciones cualitativas, permitió enfocarse en las interacciones de los actores, las cuales constituyen parte de sus activos inmateriales. A partir de ello se analizó la gobernanza del clúster turístico de Mazatlán desde dos vertientes: la que se enfoca de manera regional, y la centrada en la revitalización del centro histórico, como parte de la nueva oferta turística.

En el análisis del clúster turístico de Mazatlán se encontró que su proximidad relacional es débil, y le impide potenciar su eficiencia colectiva. Es decir, a pesar de contar con proximidad geográfica, la carencia de un dinamismo relacional entorpece otras formas de cercanía que propician la confianza entre los actores, como las proximidades social, cultural e institucional que coadyuvan al impulso de proyectos y generación de innovaciones en los espacios económicos (Boschma, 2005).

En este contexto, se considera que el clúster turístico de Mazatlán carece de un sistema de gobernanza regional dinámico, aunque existe una tendencia incipiente para trabajar de manera conjunta, pero selectiva. Por ejemplo, los actores han aprendido a gestionar en bloque, ante la administración local, para agilizar la solución de problemáticas que incidan en la industria, y que resolverlas dependa de la jurisdicción municipal. Si bien gestión colectiva es un paso adelante respecto a la posibilidad de trabajar por el destino, sobre todo si los recursos son en infraestructura urbana, el problema más acuciante es que el factor que aglutina a los actores de mayor fuerza económica y política es la gestión de recursos para promoción, y se deja de lado otro tipo de cooperación que beneficie al destino como producto turístico.

En cuanto al centro histórico, si bien está inserto en la dinámica del clúster, el origen de su sistema de gobernanza obedece al empoderamiento de grupos 
locales no ligados a la industria turística, lo que ha propiciado una organicidad territorial con capacidad de gestión tanto en su recuperación, primero como espacio urbano, y luego como parte de la oferta turística. El hecho de que el objetivo central que impulsó la gobernanza del centro histórico fuera su recuperación como lugar de vida, ha sido trascendental para su éxito pues les ha permitido el acceso a fondos gubernamentales que trascienden al sector turismo, así como la incorporación al proyecto de actores públicos y habitantes connotados de la zona.

La mayoría de los estudios de clústers parte del supuesto de que existe una sola red de gobernanza, aquí se encontró que en un mismo clúster pueden existir o emerger redes diferenciadas, elemento central para entender las contradicciones y los conflictos factibles de surgir dentro de los clústers. En general, se puede concluir que la gobernanza regional del clúster turístico de Mazatlán es débil e insuficiente, para revertir las deficiencias que ubican al destino en una posición poco competitiva en el mercado turístico nacional. En contraparte, la gobernanza intraurbana circunscrita al centro histórico, que inició como una acción ciudadana no institucionalizada, ha sido más eficiente pues logró la recuperación del casco antiguo de la ciudad, y contribuyó a dinamizar la actividad turística porque le permitió diversificar la oferta y ampliar sus nichos de mercado. Sin embargo, conforme el centro histórico ha ganado importancia turística, el sistema de gobernanza intraurbano corre peligro de ser absorbido por el regional, que ya lo ha identificado como punto estratégico del relanzamiento de Mazatlán en los mercados turísticos. El riesgo es que se retiren los individuos que consideran al centro histórico como parte fundamental de la vida de la ciudad, y no solo como un elemento de la diversificación turística, ya que, como sugieren Porter y Shaw (2009), los gobiernos tienden a impulsar políticas de revitalización urbana porque considera que los centros son espacios vacíos en los cuales pueden “dibujar” una ciudad nueva; por ello, para mantener la eficiencia del sistema intraurbano es fundamental la participación tanto de los habitantes como de las entidades económicas y gubernamentales, pues solo de esa manera será factible que el centro histórico de Mazatlán cumpla sus dos funciones cruciales: ser espacio de vida y parte fundamental de la experiencia turística.

\section{Referencias}

Alvarado, L. (2007). El centro histórico de Mazatlán: muchos espacios, muchos tiempos. En A. Lizárraga (coord), Nací de aquí muy lejos. Actores locales y turistas en el centro histórico (pp. 19-60). México: Universidad Autónoma de Sinaloa.

Azizpour, F., y Fathizadeh, F. (2016). Barriers to collaboration among tourism industry stakeholders. Case study: Mashhad Metropolis. AlmaTourism, Journal of Tourism, Culture and Territorial Development, 7(13), 48-65. doi:10.6092/issn.2036-5195/5991 
Baggio, R., Scott, N., y Cooper, C. (2010). Improving tourism destination governance: a complexity science approach. Tourism Review, 65(4), 51-60. doi: 10.1108/16605371011093863

Beaumont, N., y Dredge, D. (2010). Local tourism governance: A comparison of three network approaches. Journal of Sustainable Tourism, 18(1), 7-28. doi: 10.1080/09669580903215139

Beritelli, P., Bieger, T., y Laesser C. (2007). Destination governance: using corporate governance theories as a foundation for effective destination management. Journal of Travel Research, 46(1), 96-117. doi: $10.1177 / 0047287507302385$

Bieger, T. (1998). Reengineering destination marketing organisations: The case of Switzerland. The Tourist Review, 53(3), 4-17.

Borgatti, S., Everret, M., y Freeman, L. (2002). Ucinet for Windows: Software for Social Networks Analysis. Cambridge: Harvard University Press.

Boschma, R. (2005). Proximity and innovation: A critical assessment. Regional Studies, 39(1), 61-74. doi: 10.1080/0034340052000320887

Caffyn, A., y Jobbins, G. (2003). Governance capacity and stakeholder interactions in the development and management of coastal tourism: Examples from Morocco and Tunisia. Journal of Sustainable Tourism, 11(2), 224245. doi: 10.1080/09669580308667204

D’Angella, F., y Gob, M. F. (2009). Tale of two cities collaborative tourism marketing: towards a theory of destination stakeholder assessment. Tourism Management, 30(3), 4219-440. doi:10.1016/j.tourman.2008.07.012

Duran, C. (2013). Governance for the tourism sector and its measurement. UNWTO Statistics and TSA. (Issue Paper Series STSA/IP/2013/01). Recuperado de http://statistics.unwto.org/en/content/papers

Giuliani, E. (2010). Clusters, networks and economic development: an evolutionary economics perspective. En R. Boschma y R. Martin (Eds), The handbook of evolutionary economic geography (pp. 261-279). Reino Unido: Edward Elgar.

Gobierno del Estado de Sinaloa. (2006). Segundo informe de gobierno de Jesús Alberto Aguilar-Padilla. Culiacán, Sinaloa, México.

Gobierno del Estado de Sinaloa. (2008). Cuarto informe de gobierno de Jesús Alberto Aguilar-Padilla. Culiacán, Sinaloa, México.

Gobierno del Estado de Sinaloa. (2010). Sexto informe de gobierno de Jesús Alberto Aguilar-Padilla. Culiacán, Sinaloa, México.

Gobierno del Estado de Sinaloa. (2013). Tercer informe de gobierno de Mario López Valdez. Culiacán, Sinaloa, México.

Gobierno del Estado de Sinaloa. (2016). Sexto informe de gobierno de Mario López Valdez. Culiacán, Sinaloa, México.

Merinero, R. (2010). Desarrollo local y análisis de redes sociales: el valor de las relaciones como factor de desarrollo socioeconómico. Redes. Revista Hispana para el Análisis de Redes Sociales, 18(11), 277-304.

Merinero, R. (2015). La agenda de investigación del turismo mediante el análisis cuantitativo de redes Sociales (QSNA). Cuadernos de Turismo, 36, 269-294. doi: 10.6018/turismo.36.230991 
Nava-Zazueta, M. (2012). Encuesta sobre dinámica relacional a actores territoriales en el clúster turístico de Mazatlán.

Nava-Zazueta, M. (2013). Innovación en el territorio y capacidad competitiva de los destinos turísticos. Redes y capital territorial en Mazatlán. México: Juan Pablos y Universidad Autónoma de Sinaloa.

Nava-Zazueta, M. (2017). Des-diferenciación de los espacios del turismo en Mazatlán. La calle, el espacio público, en la construcción del producto turístico. Internacionales, Revista en Ciencias Sociales del Pacífico Mexicano, 3(5), 42-89.

Nava-Zazueta, M., e Ibarra, G. (2010). Actores locales y competitividad turística en Mazatlán. El territorio como producto turístico. Topofilia, Revista de Arquitectura, Urbanismo y Ciencias Sociales, II(1), 1-15.

Nava-Zazueta, M., y Valenzuela, B. (2014). Acción colectiva y gobernanza del centro histórico de Mazatlán, México. Recuperación y conversión a espacio. Ánfora, 21(36), 125-148.

Nordin, S., y Svensson, B. (2007). Innovative destination governance: The Swedish ski resort of Are. The International Journal of Entrepreneurship and Innovation, 8(1), 53-66. doi: 10.5367/000000007780007416

Pearce, D. G. (2014). Toward an integrative conceptual framework of destinations. Journal of Travel Research, 53(2), 141-153. doi: 10.1177/0047287513491334

Pechlaner, H., Beritelli, P., y Volgger, M. (Eds). (2015). Contemporary destination governance: A case study approach. Vol. 6. Emerald Group Publishing. doi: 10.1108/S2042-144320140000006055

Porter, L., y Shaw, K. (Eds.). (2009). Whose urban renaissance? An international comparison of urban regeneration strategies. Londres y Nueva York: Routledge Studies in Human Geography.

Prats, L., Guia, J., y Molina, F. (2008). How tourism destinations evolve: The notion of Tourism Local Innovation System. Tourism and Hospitality Research, 3(8), 178-191. doi: 10.1057/thr.2008.24

Queiroz, F., y Rastrollo-Hortillo, M. (2015). State of art in tourist destination governance. Tourism \& Management Studies, 11(2), 47-55. doi: 10.18089/ tms.2015.11206

Santamaría, A. (2007). El centro histórico de Mazatlán como oferta turística. En A. Lizárraga (coord.), Nací de aquí muy lejos. Actores locales y turistas en el centro histórico (pp. 61-120). México: Universidad Autónoma de Sinaloa.

Scott, N., Cooper, C., y Baggio, R. (2008). Destination networks. Four Australian cases. Annals of Tourism Research, 35(1), 169-188. doi: 10.1016/j. annals.2007.07.004

Secretaría de Turismo, Datatur. (2017a). Principales indicadores de operaciones de aeropuertos por centro turístico, 2006-2015. Recuperado de http:// www. datatur.sectur.gob.mx/SitePages/TrasnAerea.aspx

Secretaría de Turismo, Datatur. (2017b). Llegada de cruceros por destino, 19922016. México. Recuperado de http://www.datatur.sectur.gob.mx/SitePages/Actividades\%20en\%20Crucero.aspx 
Svensson, B., Nordin, S., y Flagestad, A. (2005). A governance perspective on destination development -exploring partnerships, clusters and innovation systems. Tourism Review, 60(2), 32-7. doi: 10.1108/eb058455

Svensson, B., Nordin, S., y Flagestad, A. (2006). Destination governance and contemporary development models. En L. Lazzeretti, L. y C. Petrillo, C. (Eds.), Tourism local systems and networking (pp.83-92). Reino Unido: Elsevier.

Wasserman, S., y Faust, K. (1994). Social network analysis. Methods and applications. Cambridge: Cambridge University Press.

Watkins, M., y Bell, B. (2002). The experience of forming business relationship in tourism. International Journal of Tourism Research, 4(1), 15-28. doi: $10.1002 / j \operatorname{tr} .337$ 